\title{
EL RETO DE LA PRÁCTICA PEDAGÓGICA EN DOCENTES Y ESTUDIANTES: SISTEMATIZACIÓN DE LA IMPLEMENTACIÓN DE UN MODELO DE ACOMPAÑAMIENTO DE PRÁCTICA EN APOYO A LA FORMACIÓN INICIAL DOCENTE
}

\author{
THE CHALLENGE OF THE PEDAGOGIC PRACTICE IN \\ TEACHERS AND STUDENTS: SYSTEMATIZE OF THE \\ IMPLEMENTATION OF A MODEL OF ACCOMPANIMENT \\ OF PRACTICE IN SUPPORT TO INITIAL EDUCATIONAL \\ FORMATION
}

Viviana Soto Aranda

Pedagoga en Educación Parvularia (PUC) Mg. en Educación (UMCE) Mg. en Sociología (U. de Chile)

Diplomada en Asesoría pedagógica (PUC)

Diplomada en Tutoría Virtual (UAHC)

Especialista en asesoría curricular y redes de procesos educativos participativos con organismos comunitarios e instituciones educativas.

Universidad de Chile Ignacio Carrera Pinto 1045 viviana.soto.a@uchile.cl

Resumen: El artículo presenta la sistematización a la aplicación del Modelo de acompañamiento a la práctica profesional de veinte estudiantes de la carrera de Educación Parvularia y Básica Inicial de la Universidad de Chile en el desarrollo de la práctica pedagógica situada en diversos contextos educativos. El modelo ha implicado en la formación de los estudiantes y develado como sus tres elementos fundamentales: la reflexión, diálogo y colaboración han sido sustanciales para la acción pedagógica con niños, niñas y adultos; a su vez cómo los ámbitos constituyentes del acompañamiento han visto 
favorecida la acción participativa, la crítica y el análisis sistemático del contexto y la promoción de un vínculo de saber pedagógico con las instituciones educativas.

Palabras claves: sistematización, acompañamiento, práctica pedagógica, docentes, asignatura de práctica.

Abstract: The article presents the systematizing to the implementation of the Model of accompaniment to the professional practice of twenty students of the career of initial basic early childhood and initial education of the Universidad de Chile in the development of the pedagogic practice located in educational diverse contexts. The model has implied in the formation of the students and unveiled as its three fundamental elements: the reflection, dialog and collaboration have been substantial for the pedagogic action with children and adults; at the same time how the areas constituting the accompaniment have been favored the participative action, criticism and the systematic analysis of the context and the promotion of a link of pedagogical knowledge with the educational institutions.

Key words: Systematization, accompaniment, pedagogic practice, teachers, subject of practice.

\section{INTRODUCCIÓN}

Los desafíos en la educación atraviesan todos los niveles de enseñanza. La universidad en su rol formador le compete asumir de manera responsable esta tarea. Esto implica una revisión y la promoción de procesos y metodologías formativas que articulen los diversos campos del conocimiento. Este contexto nos lleva a emprender diversas acciones que debemos mirar como una gran oportunidad de organización académica.

El carácter práctico de la formación en pedagogía cobra relevancia, porque siendo un eje formativo, es la actividad que permite dialogar con la experiencia y la teoría, transformándose en una oportunidad real para ampliar $\mathrm{y}$ fortalecer aprendizajes en los estudiantes. Este énfasis requerirá de esfuerzos que signifiquen construir bases sólidas para un aprendizaje amplio a partir de un enfoque de 
diálogo y colaboración al interior de las carreras como en las relaciones que establezcan la universidad con los centros de práctica. Esta construcción pretende ser un aporte sustantivo a la reflexión, a la acción y toma de decisiones relevando el sentido curricular del proceso de la práctica y su fundamentación como proceso y creación de conocimientos en redes de colaboración.

A partir de lo anterior, la búsqueda de una sustantiva práctica pedagógica ${ }^{1}$ de los estudiantes surge de la convicción que es necesario y fundamental resguardar la calidad de la formación académica, construir y consolidar redes y vínculos en perspectiva para trabajos colaborativos en reciprocidad con instituciones que tienen la responsabilidad de la educación de niños y niñas. Esto constituye un elemento clave considerando el desafío país por una mejor educación. Por consiguiente, una nueva modalidad en el proceso de la práctica de estudiantes en una carrera de pedagogía aportará importantes insumos para fortalecer la docencia en la educación superior y para iniciativas de la institucionalidad en el área de educación de la Universidad de Chile.

Este trabajo es una sistematización al análisis de la práctica pedagógica de veinte estudiantes en práctica profesional de la carrera de Pedagogía en Educación Parvularia y básica inicial durante el segundo semestre del año 2014 e inicios del año 2015, el cual recoge sus reflexiones y análisis de la praxis a partir de implementación del modelo de acompañamiento en la asignatura de práctica de la carrera. El trabajo explicita de manera sucinta el acompañamiento a la praxis pedagógica con $\operatorname{los}^{2}$ estudiantes, para dar paso a los significados de su implementación en un inicial ejercicio docente.

\section{EL MODELO DE ACOMPAÑAMIENTO DE PRÁCTICA A ESTUDIANTES: UN NUEVO ENFOQUE A LA PRAXIS PEDAGÓGICA}

\section{Relación dialógica de la triada pedagógica constituida por la Institución Universitaria, institución Educativa y el estudiante}

En esto se configura la relación de triada, que parte del enfoque de una relación de una acción con otros. Esta dimensión tiene como 
objetivo desarrollar un diálogo cercano, profundo y crítico, que consiste en la relación dialógica entre tres actores partícipes de una práctica pedagógica que conversan, transparentan sus posiciones, expectativas y evalúan el proceso desarrollado, siendo el protagonista central, el estudiante. Se intenciona esta relación para contribuir, a partir del contexto y situación real, su aprendizaje. El diálogo, la crítica y autocrítica constituyen elementos importantes y permiten retroalimentar de manera amplia y nutrida a partir de la experiencia educativa. El diálogo entre escuela o centro infantil, estudiante y universidad (que se traduce en el docente guía ${ }^{3}$ del centro de práctica, la estudiante y docentetutor $^{4}$ de la universidad) se promueve de manera permanente considerando en esta relación, la evaluación del proceso de la praxis desarrollada por el estudiante, implicando en esta relación la retroalimentación y autoevaluación.

\section{El Acompañamiento en el aula}

Se entenderá como un proceso que busca mediar y potenciar "in situ" aprendizajes durante el desarrollo de la práctica pedagógica del estudiante. En esta relación se potencia la colaboración entre docente y estudiante en contexto pedagógico real. Se considera relevante resituar lo que el estudiante sabe, y movilizarlo para orientar sus percepciones, facilitando de este modo, sus aprendizajes. Esta dimensión pretende a su vez considerar la relación del tutor ${ }^{5}$ con el estudiante, mediante el apoyo y colaboración constante, esto porque se concibe, a partir de este modelo, que los momentos y espacios destinados al desarrollo de la praxis serán de observación, interacción y comunicación; un fortalecimiento del proceso de enseñanza y aprendizaje tanto para la tutoría, como para el estudiante.

El acompañamiento es el que sostiene y potencia el trabajo del tutor, de este modo, busca dar espacios presenciales y virtuales para el apoyo del trabajo pedagógico (con materiales como documentos, bibliografías, preguntas, reflexiones en torno a las propuestas educativas que se programan). Es reconocer y dar sentido a un estar consciente con el otro, en palabra de Paulo Freire quien nos señala: "El hombre heredando la experiencia adquirida, creando y recreando, integrándose a las condiciones de su contexto, respondiendo a sus desafíos, 
objetivándose así mismo, discerniendo, trascendiendo, se lanza a un dominio que le es exclusivo: el de la historia y el de la cultura" (1979:31); de este modo, el hombre al estar en el mundo y con el mundo se conecta $\mathrm{y}$ se relaciona desde otra perspectiva que lo invita a ser actuante y consciente.

\section{Aprendizaje colaborativo entre pares}

Se entenderá como la interacción entre estudiantes que, en el contexto de prácticas, siendo de igual o distinto nivel formativo en la carrera se acompañan en su ejercicio docente. El aprendizaje colaborativo es un elemento esencial que contribuye al concepto de acompañamiento, el colaborar implica estar con el otro, dialogar, escuchar, y participar en acciones conjuntas. Esta dimensión fomenta el desarrollo de metodologías cooperativas y el desarrollo de competencias profesionales; como a su vez, el desarrollo de estrategias de comunicación y de trabajo en equipo. Las ventajas son reconocidas (Solsona, 1999, Montero, 2001) ya que este tipo de aprendizaje desafía y permite poner en juego todas las habilidades para el logro de objetivos, facilitando la ampliación y el fortalecimiento del saber docente.

\section{Diversidad de contextos, localidades y tipos de establecimientos para ampliar y potenciar el conocimiento durante la práctica}

Centros de prácticas son un desafío, Valle y Manso, (2011). El diverso contexto educativo, social, cultural cobra sentido como espacio para la colaboración y aprendizaje. Esta dimensión permite rescatar la pertinencia y promover acciones que emerjan de la comunidad y la experiencia de esta diversidad provee un escenario de oportunidades en la construcción de conocimiento que invite a desafiar a los estudiantes y a la docencia. Esta dimensión implica favorecer relaciones colaborativas que permita un desarrollo armónico de la práctica pedagógica en los diferentes espacios y ambientes educativos en pertinencia con la cultura de cada centro, en aporte al conocimiento de las comunidades educativas y sus participantes. Esta dimensión busca diversificar los centros de práctica dando la posibilidad de enriquecer el trabajo formativo docente, 
aprender de dichas realidades y colaborar de manera conjunta en mejoras educativas. Del mismo modo, insta a ampliar redes en contextos diversos ya sea rurales y urbanos, y centros en diversas instituciones y modalidades curriculares de enseñanza.

\section{La búsqueda del conocimiento a partir de lo que se está haciendo resulta un eje articulador de mejoras}

Esta dimensión considera la importancia de observar la realidad, de manera crítica hacer una lectura del contexto, develar preguntas que nos lleven a indagar, analizar y proponer acciones para el desarrollo de la formación docente y la práctica pedagógica y relevar con ello, los procesos de reflexión en la acción. Para esto se busca intencionar el trabajo conjunto entre docentes que busquen y promuevan la investigación en temáticas específicas en el desarrollo de la práctica pedagógica. A partir del proceso vivido en la acción docente, la tutoría de la universidad y estudiantes buscarán significar la experiencia, los hechos que acontecen, recoger información y analizarla; lo que responde a visibilizar lo aprendido, las acciones, tensiones, y desafíos que en la fase de práctica se presentan. El objetivo es, a partir de la experiencia en el contexto de la práctica de estudiantes, generar y difundir el conocimiento en beneficio de los aprendizajes de las comunidades e instituciones educativas escolares y de educación superior. Esta dimensión pretende generar productos de investigación de los estudiantes y de la docencia en esta materia, que permitan contribuir ampliamente al conocimiento y la promoción de iniciativas a la formación docente.

\section{SISTEMATIZACIÓN DEL ACOMPAÑAMIENTO A LA PRÁCTICA PEDAGÓGICA}

Para diversos autores, (Freire, 1971, Schön, 1983; Perrenaud, 2007; Litwin, 2008) resulta necesario la reflexión acerca de lo que se hace y lo que se ha hecho, ambas son condiciones necesarias por sobre todo en el educar. Por cierto, la reflexión permite enfrentar diversas situaciones que se presentan en el contexto escolar. 
Considerando su pertinencia, cabe dar relevancia a la sistematización de la experiencia, que según diversos autores (Jara 1994, Martinic, 1998, Ghiso, 1998, Garcia - Huidobro 1998, De Sousa 2000, Torres, 2003; Messina, 2004) distinguen de manera crítica, la experiencia y los significados de ella, su acontecer; para comprenderla y promover un nuevo conocimiento.

La sistematización no es un concepto univoco, éste es entendido como la mirada crítica que empleamos sobre una experiencia y sus procesos. Es la primera teorización sobre esta en la que se cuestiona, se relaciona permitiendo un análisis más profundo que posibilite transformaciones sobre lo que se hace, para la incorporación de cambios de manera de enriquecerla. La sistematización señala Ghiso (1998), vincula un componente que es el pedagógico, que nos formamos para sistematizar y al sistematizar nos estamos formando. La sistematización pretende explicar el significado que ha tenido la experiencia para los diferentes participantes y constituirse en un nuevo conocimiento. El sustento epistemológico que subyace en esta sistematización es que los sujetos en su devenir van construyendo realidades en una dimensión objetiva y subjetiva, inmersos en un proceso histórico de configuración y comprensión de fenómenos individuales y colectivos; sociales y educativos; dinámicos y complejos. De este modo, la sistematización desde un enfoque interpretativo crítico da cuenta de aspectos metodológicos que implican reunirse con los participantes de la experiencia para reconstruir, interpretar y teorizar surgiendo como un carácter formador y transformador de la experiencia.

\section{EJE DE LA SISTEMATIZACIÓN. ¿QUÉ Y CÓMO SE SISTEMATIZÓ?}

Interesa la experiencia de lo que ha sido para los estudiantes realizar su práctica profesional en centros educativos, con el énfasis de rescatar el contexto de la praxis y reflexionar sobre el hacer pedagógico e indagar en él, para promover nuevos conocimientos. Este propósito buscó responder la pregunta que convocó sistematizar: ¿Qué significado otorgaban los estudiantes de la Carrera de Educación Parvularia y Básica inicial al modelo de acompañamiento?, siendo el objetivo: Comprender que elementos distintivos develan los estudiantes en 
práctica profesional a su praxis pedagógica desde la perspectiva del enfoque y elementos constituyentes del modelo de acompañamiento. Para sistematizar se consideró rescatar la experiencia al término del proceso de práctica, esto es, a finales del 2014 e inicios del año 2015. Es así que al finalizar el proceso se recurre a dos instancias: una evaluación grupal a veinte estudiantes y la recopilación de información de sus análisis durante desarrollo de su praxis pedagógica. Con base en la lectura de los relatos, se pasa a la segunda fase de análisis e interpretación del conjunto de las experiencias en relación a la implementación del modelo de acompañamiento. En esta fase se busca el sentido socialmente construido de la experiencia y producir una interpretación desde los referentes conceptuales pertinentes a la investigación implícita en los elementos o dimensiones constituyentes del modelo. Es así, como se analizó la experiencia vivida, no sólo en función de los aspectos y relaciones que sus actores consideraran relevantes, sino también, desde las categorías iniciales de análisis sustentadas en las cinco dimensiones del modelo de acompañamiento, tales como: triada pedagógica, acompañamiento, aprendizajes entre pares, la pertinencia de los contextos y la reflexión e investigación.

\section{SU ANÁLISIS}

La asignatura de práctica en la carrera cobra muchos desafíos para el estudiante, y docente que realiza tutoría a estudiantes en práctica, implicando diversos ámbitos de abordaje en su desarrollo y discusión. En este sentido, la tutoría en el modelo de acompañamiento busca acompañar en un saber estar con los participantes del centro educativo, buscar encuentros y dialogar con el estudiante en apoyo al trabajo pedagógico con los niños y niñas. De tal modo, se ha podido rescatar que la tutoría cobra relevancia y tiene un alto significado en las prácticas. La tutoría de la Universidad es aporte indispensable para la relación que establece el estudiante con el centro de práctica. Expresiones de las protagonistas rescatan el acompañamiento en el proceso formativo relevando el apoyo pedagógico necesario para enfrentar un contexto diverso y complejo como lo es la escuela. Al respecto una estudiante expresa: 
...deja un precedente positivo respecto al complejo proceso de práctica profesional... La tutoría ha demostrado compromiso y disposición. $\mathrm{Y}$ es importante considerar que el tutor de la Universidad es un apoyo en el proceso de práctica al inicio, durante y al término de ésta. La disposición de la tutoría en el centro de práctica (epp). ${ }^{6}$

Se devela el reconocimiento del apoyo de la tutoría durante el proceso de aprendizaje de la estudiante. La presencia de la tutora en el centro representa un importante apoyo para la estudiante, y para el logro de su tarea educativa. El ejercicio tutorial se reviste de un carácter relacional y es lo que sustentaría a la denominada tutoría implicada en el modelo de acompañamiento que se caracteriza principalmente en: acoger, tener apertura al otro, dialogar, participar "con", facilitar y orientar el trabajo pedagógico. Al respecto una estudiante expresa lo siguiente:

...en este proceso no estuve sola, fue parte importante mi tutora de la universidad, fue un apoyo y pilar fundamental, orientándome siempre que lo necesité y en las ocasiones que requerí de ella, de su apoyo o consejo, creo que, sin este apoyo tan importante, el desarrollo de esta práctica hubiera sido mucho más tensionante. (epp).

Se significa la experiencia pedagógica, un aliciente gatillador en la formación docente. Se posiciona al tutor como un otro embestido de significados que aporta de manera sustantiva a la formación docente. En la relación estudiante y tutoría no estuvo exenta de dificultades, esto por lo que representa ser acompañado por un tutor que observa y evalúa a la vez; dicha situación se fue trabajando con el estudiante de modo construir aperturas para una relación en colaboración. Estas situaciones develaron un iniciar lento del proceso de la praxis de los estudiantes, por temor a equivocarse, por lo nuevo, por lo desafiante en términos de exigencia y compromisos consigo mismo, con el centro y con los niños y niñas y la universidad. La tutoría acompaña, observa y participa siendo parte del equipo guía del centro de práctica facilitando la construcción de aprendizaje en forma conjunta y colaborativa. Por tanto, la tutoría, su forma de relacionarse, lo que infeccionará y cómo; serán decisivos para los aprendizajes del estudiante. 
La experiencia ha significado relevar la retroalimentación como un acto pedagógico esencial para el aprendizaje, no sólo del estudiante sino también para quienes ejercen la docencia. La retroalimentación se considera permanente y a partir de distintas modalidades, ya sea presencial, en espacios áulicos de la universidad, en los centros de práctica, en conversaciones e instancias de evaluación, y en modalidad virtual, con envíos de análisis de material educativo. Estudiantes rescatan la importancia del apoyo pedagógico y develan del acompañamiento, un compromiso docente, así lo expresan:

Se demuestra disposición e interés por el trabajo en la práctica y ha habido retroalimentación que es una gran ayuda. Esto proporciona que las estudiantes tengan una actitud positiva hacia el docente, sentir seguridad y confianza, facilitado la realización un buen trabajo en la escuela. (epp).

El acompañamiento implica un proceso de tutoría integral, en este sentido releva los saberes y el con-vivir en la formación de los estudiantes. El proceso de acompañamiento es relevado porque permite hacer parte a la tutoría de su propia experiencia de aprendizaje y ésta, rescatar la experiencia de práctica en contexto real con todas sus complejidades; de este modo implica comprender mejor la realidad. Se destaca como bien señalan estudiantes: "esta forma de acompañamiento, [...] nos otorgó un seguimiento positivo y se contó con apoyo real de docentes para contribuir al trabajo en aula”. (epp).

Otro estudiante detalla:

Contribuye a sentirse más acompañada por la Universidad y contribuye a generar lazos con los profesionales de la escuela y con el equipo de práctica de la Universidad. Así es como rescato la preocupación para que las estudiantes realmente tuvieran una experiencia significativa que nos motivara a seguir y nos posicionara en nuestro rol como educadores. (epp).

Las vivencias que se presentan en el acompañamiento son variadas; a propósito, estudiantes manifiestan expresar sus necesidades sin dificultad. Esta distinción y apoyo en lo profesional y emocional hacia el estudiante, resulta ser preciso y vital para un proceso formativo docente y el ejercicio profesional comprometido por y con los niños, niñas y adultos. 
En relación, se puede develar que un elemento distintivo y simbólico en el acompañamiento fue la confianza que se depositó en el estudiante para su ejercicio docente, lo que resultó significativo porque permitió en ellos, creer en sus propias capacidades. Un elemento fundamental es que la tutoría posiciona el diálogo en la praxis de la estudiante lo que promovió acercamientos y construcción de vínculos, en este sentido, vitales para el ejercicio de un saber pedagógico colectivo.

La colaboración y el diálogo resultan ser gatillantes. El diálogo es valorado en la práctica, se desafía al estudiante, y les impulsa a movilizar sus saberes y las experiencias, comunicándolas a los otros. Como señala una estudiante, “... se valora [el diálogo] por la importancia que esto tiene en la formación de un docente, se promueve valores que sabrá posteriormente trasmitirse en la enseñanza". El diálogo es reconocido como elemento sustantivo en la formación y ejercicio docente, lo que aporta a la experiencia resulta significativo. La colaboración implicó estar comprometido con la acción del otro, de aportar con ideas al material pedagógico. La colaboración de la tutoría en el centro implicó que las relaciones en y de los estudiantes fluyeran y se participara de manera más activa en aporte a su praxis.

El acompañamiento ha resultado ser el punto de partida de la práctica pedagógica por estar significando la praxis. En este sentido, y desde el modelo de acompañamiento, observar el proceso pedagógico conlleva dos miradas que se complementan, una, por un lado, resguardar que el otro a quien se observa no se sienta invadido y con ello el espacio en el que participa no se vea interferido, y otra, un observar participando implica un hacer compartido y saber que el otro es parte activa de tu experiencia. De este modo, se busca relevar el proceso pedagógico que realiza el estudiante con un estar en diálogo con, para aportar al aprendizaje del estudiante con herramientas para una apuesta didáctica consciente y autónoma.

El enfoque dialógico del acompañamiento permite develar que los espacios y momentos al diálogo resultan diversos y complejos, esta notoriedad se presenta y se logra constatar cuando se invita a la participación activa de los niños y niñas durante la práctica pedagógica. Este enfoque interpela nuestra propia praxis tanto de la tutoría, de la docente guía y del estudiante. El diálogo implica disposición a otros, porque requiere de escuchas, de aceptación en esa atención al otro; el diálogo es la posibilidad de ir objetivando una propuesta pedagógica, 
esto implica apuestas y propuestas en aceptación. Las tensiones se hacen presentes por las barreras de lo instituido en las comunidades educativas. Lo complejo se hace evidente y el proceso participativo se torna lento, esto insta a un volver la mirada sobre lo que se hace y como se hace, dar una vuelta de mirada al contexto y su hacer para poder comprender $\mathrm{y}$, de esta forma, volver a situarse comprendiendo más y mejor. En ese hacer comprensivo se busca desplegar conversaciones e invitaciones para un trabajo colectivo, pero, explicarlo con el sentido del reconocimiento de los otros para la acción pedagógica. Este diálogo implica motivaciones para los estudiantes, implica asumirse en su rol pedagógico, y les desafía desplegar habilidades y conocimiento.

Estudiantes dan relevancia a la relación entre la universidad y el centro de práctica, esta les ha contribuido a interactuar sobre acciones colaborativas institucionales, a desarrollar proyectos participativos, a promover colaboración y de este modo, generar más aprendizaje. Este ejercicio de institucionalidad promueve la articulación de ideas, y acciones como espacios de aprendizaje colectivo real.

Se puede decir que el acompañamiento implicó estar en sintonía con el sujeto de la experiencia y el contexto. A su vez, implicó develar las potencialidades y las dificultades presentadas por los estudiantes; las que se fueron conversando y reconociendo de manera constante. Las necesidades expresadas por los estudiantes referían principalmente a la atención personalizada hacia los niños y niñas, lo que daba cuenta de las múltiples acciones que demanda la escuela y centros infantiles donde los aportes son necesarios y requeridos. Esto permitió que se conversara in situ sobre la experiencia, su acontecer estaba tensionado por el tiempo que disponía el estudiante en el centro en relación a las demandas que se solicitaban en el quehacer con los niños y docentes; es decir los estudiantes en el centro entraban en una dinámica de múltiples actividades y el tiempo para la pausa, para la conversación se hacía acotado. El tiempo resulta ser vital y la planeación y organización para la conversación resulta crucial. El acompañamiento, en este sentido, se fue haciendo más necesario y urgente con mayor permanencia en el centro y planeando un trabajo más colaborativo. Un aspecto importante es que los centros de práctica están atentos a nuestro trabajo pedagógico y colaboración, lo que demanda estar en sintonía con su contexto y posibilidades de acción. 
La relación en una triada se hace más evidente en la evaluación que se realiza al estudiante como parte de procedimientos de seguimiento al trabajo pedagógico. En este sentido se expresa.

Se releva la evaluación en triada, representa una muy buena instancia en el que tanto la educadora como la tutora de práctica pueden evaluar constructivamente a la estudiante, haciendo conocer todas las debilidades y fortalezas siendo una buena herramienta para nuestro desempeño como estudiantes en práctica y futuras educadoras. (epp).

La evaluación en triada se logra visibilizar como apoyo pedagógico, "ayuda a la retroalimentación constante, se podía dialogar en torno a la práctica", señala un estudiante; relevándola como recurso para reflexionar con otros. De esta manera se valora establecer vínculos con la escuela, que permita promover acciones concretas de diálogo y trabajo conjunto, por sobre todo con un actor clave como es el docente guía, con quien trabaja de manera directa el estudiante en práctica, siendo el profesional el cual que guía, dialoga y colabora en el desarrollo profesional e inserción del estudiante en la institución, centro de práctica.

El acompañamiento implica acciones y frecuencias, la asistencia al centro de práctica, se programa con la estudiante para organizar el trabajo y dicha definición del acompañamiento implica que el propósito de este no es interferir, sino ser parte de una acción pedagógica. Al respecto los estudiantes si bien relevan la presencia participativa de la tutoría, dan cuenta de dificultades referidas a las distintas formas de tutoría que se dan entre las docentes, que difieren del tiempo destinado en el centro de práctica y la cercanía en la acción pedagógica hacia el trabajo del estudiante. Este aspecto apunta a cautelar en el trabajo tutorial los criterios de acompañar; hacer claro el desafío de asumir y hacer consciente y critico un nuevo enfoque para la asignatura de prácticas, esto hace visible la tensión de lo que es una supervisión caracterizada por una observación más distante, a un acompañamiento al estudiante más colaborador y dialogante. Este es el desafío y requerirá su atención para dejar atrás al supervisor de "mirada vigilante", y dar paso a un docente tutor que participa observando, guiando y apoyando al estudiante. El acompañamiento abre la necesidad de ampliar espacios de dialogo y reflexión, esto implica que la tutoría dialogue más y reflexione sobre lo que se hace y como lo hace. 
Con los relatos de la experiencia se distingue un elemento clave que interviene en el qué y en el cómo de la propuesta, y es la concepción que se tiene del aprendizaje. De cómo se concibe que el estudiante aprenda, esto es el punto base de la propuesta. Pareciera obvio que al pensar las praxis nuestra concepción de cómo se aprende es en la acción; cabe sin embargo agregar o más bien insistir en esa acción, que se puede expresar como el ya nombrado, pero a veces poco practicado aprender haciendo. El rol activo es el que debemos sostener a favor de los niños y para facilitar el aprendizaje, esto implica pensar en su propuesta didáctica desde la acción de los niños y niñas, pensar en el que haremos de modo de vivir juntos la experiencia; promoviendo de esta manera la observación, el análisis, la pregunta por los hechos y buscar a partir de ese ejercicio, un hacer creativo. Es el contexto, en esta fase el que se releva, es importante por tanto saber reconocerlo para situarse bien en él y recoger aprendizajes abiertos y flexibles, aprendizaje en contexto real, como señala Zeichner (2010) distintos tal vez de los que se puedan dar en contextos áulicos universitarios, esto implica relevar los espacios educativos reales con adultos, niños y niñas.

$\mathrm{Al}$ respecto, la diversidad y relevancia del contexto ha sido un elemento que ha enriquecido y potenciado las prácticas pedagógicas, pues ha significado un constante desafío frente a lo diverso de los contextos sociales, diversos sujetos, espacios y culturas. Ha significado una oportunidad potenciar el diálogo con docentes, estudiantes y familias de diversas localidades y espacios educativos por las nuevas posibilidades de acción, creación, participación y aprendizajes. Cada centro es reconocido en su singularidad, por lo que el desafío ha sido constante y ha potenciado capacidades y habilidades en estudiantes como adaptarse a lo diverso, a lo nuevo, a conocer otra cultura local, establecer una relación dialógica y de trabajo colaborativo no solo con equipos de profesionales, sino también con otros actores de la comunidad. Al respecto un estudiante afirma:

La práctica pedagógica es la base de los aprendizajes que se han obtenido. La experiencia adquirida en la sexta región (en localidad rural) propició fortalecer los conceptos problematizados durante el desarrollo de la carrera y ayuda a comprender el impacto del trabajo que realiza el docente dentro de la sociedad. (epp). 
Los espacios educativos se develan como campos específicos de acción pedagógica y social a partir de los cuales se desarrollan construcciones sociales, que institucionalizan el actuar de los sujetos, por ende, el estar implicado en el contexto y comprenderlo implica apertura de acción, reflexión y un hacer consciente. El conocer esta diversidad nos amplía el conocimiento y nos permite crear herramientas para su abordaje.

El acompañamiento a la praxis logra una confluencia entre instituciones: la universidad y el centro educativo, lo que ha ido dando un carácter distintivo a la práctica. Esto porque el trabajo colaborativo y compartido no sólo se presenta en retroalimentar a estudiantes, sino también en la relación profesional en el centro de práctica. Este aspecto queda plasmado en acciones como el acompañamiento de la tutoría de la universidad al estudiante en los centros de práctica, los diálogos con profesores y educadores guías en instancias de experiencias pedagógicas, $y$ en respectivas evaluaciones, en reuniones $y$, seminarios de encuentros pedagógicos.

Estos encuentros han facilitado el desarrollo de proyectos educativos al interior de los centros de práctica, y ha permitido situar el aprendizaje, es decir, construirlo y potenciarlo a partir de un acontecer real con el conocimiento de una realidad social, educativa específica y concreta, lo que ha implicado abrir nuevas posibilidades de acción pedagógica facilitando aprendizaje en los participantes. En este sentido, resulta vital crear y trabajar los vínculos entre la Universidad e instituciones educativas, sociales y gubernamentales, que permitan promover, la construcción de redes colaborativas de trabajo y promoción conjunta de generación de conocimiento en pos de la educación de nuestros niños y niñas y comunidades educativas. Se identifica así que esta colaboración compartida sea un ejercicio político permanente para una necesaria institucionalidad en favor de nuestra sociedad.

En esta línea, el Aprendizaje entre pares que ha posicionado el modelo de acompañamiento ha contribuido a que estudiantes de práctica logren desarrollar vínculos de cooperación directa entre sí, que favorezca la construcción de conocimiento pedagógico en un mismo espacio educativo. El trabajo entre pares ha sido una instancia donde los estudiantes comparten experiencias y colaboran en la mejora de sus prácticas pedagógicas, lo que fomenta el apoyo profesional, la 
socialización y la construcción colectiva de experiencias pedagógicas. Sobre el acompañamiento entre pares estudiantes expresan:

Todo lo que uno aprende ha sido con ayuda de los profesores, pero más sin tus compañeras con las que uno comparte en la universidad y que, mejor que sean las que comparten contigo en la práctica sabiendo que es un momento que es complejo por todas las exigencias que te demandan los niños y eso que requiere de apoyos. [...] Nunca había sentido tanto entusiasmo como ahora que con mi compañera hacemos trabajo juntas, conversamos y nos organizamos con las actividades (epp).

La colaboración entre estudiantes ha sido un gran aporte para los que cursan distintos o el mismo nivel de la carrera. Así mismo, el análisis crítico, reflexivo y constructivo por parte de los estudiantes promueve el reconocimiento de fortalezas, debilidades y necesidades que permiten por medio del diálogo, el complemento de saberes en los distintos espacios de trabajo pedagógico. El aprendizaje entre pares facilita desarrollar confianza y fortalecer aprendizajes previos. El aprendizaje entre pares se ha vivido con sentido pedagógico, lo que resulta significativo por parte de quienes lo vivieron.

Esta dimensión ha contribuido a relevar la colaboración y la cooperación en la docencia, un elemento distintivo que fortalece la formación docente. Que mejor que estudiantes en formación den inicio desde sus experiencias prácticas un ejemplo de pedagogía colaborativa, este ejercicio es necesario y urgente porque implica fortalecer justamente la docencia y ampliar en ella colectivos de acción para un propósito común que es construir conocimiento participativo, con niños, niñas y jóvenes, lo que se traduce en un ejercicio de ciudadanía.

Un elemento complementario y que se devela a partir del ejercicio de colaboración y diálogos ha sido integrar el trabajo de otras asignaturas al proceso de la praxis pedagógica. En el trabajo tutorial se ha intencionado que las experiencias pedagógicas en el aula se vayan articulando con otras asignaturas de la malla curricular de la carrera. Esta modalidad de trabajo ha fortalecido los aprendizajes teóricos y prácticos en situaciones reales, dando la posibilidad de que las unidades de contenido en el trabajo académico tengan un sentido práctico y significativo para las estudiantes. Esta integración y articulación curricular ha favorecido un trabajo sistemático entre la universidad y la escuela, lo que favorece la construcción de conocimientos nuevos y la posibilidad de actualizar y flexibilizar los quehaceres pedagógicos. El 
conectar puentes con otras asignaturas contribuye la articulación de las experiencias que llevan a cabo las estudiantes en los diversos contextos educativos.

La práctica de los estudiantes de pedagogía pretende ser construidas desde una búsqueda y valoración al desarrollo de diversas acciones del trabajo docente, con un enfoque dialógico que lo promueva y posibilite impulsar los diversos procesos pedagógicos mediante la integración de saberes con el propósito de hacer conexiones del conocimiento teórico y práctico. Con esto se busca que la práctica en sí misma constituya un elemento no independiente dentro de la formación de la carrera, por ende, en diálogo con y entre las asignaturas. Este énfasis dialógico y de colaboración es lo que intenciona el modelo para el desarrollo de una práctica pedagógica vitalizada y sostenida en saber pedagógico.

La pretensión es una articulación entre diversas áreas de la docencia y, que, junto a la ampliación de conocimiento, así reconocida por estudiantes, requerirá de procesos de reflexión e investigación sobre lo que se hace. En un primer momento los procesos de reflexión cobran relevancia en tanto nos ofrecen posibilidades para el cambio y la transformación de prácticas en pos de relevar al sujeto en tanto estudiante y docente, esto es hacer consciente y crítica su subjetividad y posibilidad de hacer y hacerse con otros. Estos procesos de reflexión nos inquietan, porque aportan información y ayudan a conocer la realidad, ver con otros ojos lo que se hace. Los momentos pedagógicos se enriquecen al ser reflexionados y el docente se humaniza, parafraseando a Paulo Freire. Los momentos de reflexión deben ser permanentes, pero como dice Dewey (1998), cobran intensidad, éste nos recuerda que la reflexión suele iniciar al enfrentar situaciones complejas y hay disposición a su exploración, de esta instancia es la posibilidad también para escenarios posibles de indagación. Para estudiantes resulta significativo, así es expresado:

...seguir resaltando la importancia que tiene la observación de sí mismos como del entorno. Volver la mirada hacia nosotros es un acto de valentía y humildad para seguir mejorando. Esto sin duda repercutirá en una observación mucho más transparente con el medio, pudiendo detectar lo importante a tiempo. El realizar este análisis es justamente eso, observarme, observar lo 
que me rodea, lo aprendido, etc. Y desde ahí construir nuevos conocimientos (epp).

La práctica es un espacio en el que cohabitan diversas tensiones, propuestas y oportunidades de colaboración; y la indagación situada en contextos diversos, permitirá revelar un variado reconocimiento de los saberes y la construcción de otros nuevos. Se reconoce que la investigación como elemento vital para la promoción de conocimiento estuvo en tensión por factores de tiempo de los estudiantes en la cual, y como ya se mencionó, la práctica pedagógica en los centros educativos es múltiple, la acción educativa va demandando acciones diversas, esto dificulta muchas veces hacer pausas y hacer un seguimiento al proceso. Esto implica un análisis mayor que aquí no se podrá ampliar, pero los centros educativos para los estudiantes están siendo demandados de tal manera por el sistema educativo con énfasis en metas y pruebas de mediciones que han limitado los espacios para conversar, planear, y establecer mecanismo de indagación, a su vez, cuando la práctica en el centro no está instalada, se dificulta aún más. Hay una cultura en la escuela y en centros infantiles y requiere ser conocida para su mayor comprensión y abordaje de propuestas. Esto nos lleva a reflexionar y los análisis de los estudiantes van en esa línea de tensión y problematización.

Por lo anterior esta modalidad nos releva la importancia que reviste el diálogo, los encuentros profesionales, las interacciones, la crítica en el proceso formativo y en el hacer pedagógico. La práctica docente se ve implicada en un constructo permanente.

Para esto, es necesario un saber estar en el contexto educativo real con énfasis en la colaboración y el diálogo con los otros. La experiencia pedagógica en un contexto es la práctica situada y auténtica que posibilita la ampliación y profundización del conocimiento. Se enfatiza en la propuesta, la experiencia y el desafío de ser un conector relevante y constructivamente significativo para el aprendizaje. Este saber estar implica situarse observante, reflexivo y crítico. Lo que vuelve más significativa y relevante la enseñanza nos dice Paulo Freire, parece ser esa conexión y las relaciones con el mundo y para el mundo. Al respecto este estar se expresa en la relevancia al sujeto estudiante, hace sentido y se extrapola al niño y niña. El saber estar se hace compartido, esa es nuestra apuesta del acompañamiento, esa debe ser nuestra pedagogía. El estar es con, eso hace que vivamos la experiencia con un sentido 
realmente compartido y situado desde y con otros. Al respecto una estudiante expresa:

En mi experiencia, logré posicionarme desde un rol mucho más profesional [...] Me sentí muy segura de mi formación y de los ideales en torno a la educación que quería transmitir. Creo que eso se vio reflejado en el vínculo generado con la escuela y con sus miembros, desde los auxiliares hasta el Director (epp).

El modelo de acompañamiento releva el vínculo y los vínculos, lo que nos hace conscientes de otros, que no estamos solos y que cada uno importa y aporta. El hacer pedagogía implica desafiarnos y hacernos conscientes y críticos de nuestras actuaciones, posibilidades y consecuencias; implica hacernos cargo de nuestros trayectos de vida.

Por ello, el estar con, sea un eje de reflexión y análisis al inicio y durante el proceso de acompañamiento de la praxis pedagógica de manera permanente, porque hoy en nuestra sociedad no es habitual estar acompañado, la lógica individualista y competitiva, ha coaptado nuestro mundo de la vida. Lo que nos cabe es seguir reflexionando y analizando lo realizado, lo aprendido y lo que nos queda por hacer, como seguir sistematizando la acción cada vez con más participantes, para hacerla ampliamente crítica y por ende con mayor sentido.

\section{REFLEXIONES FINALES}

La evidencia nos aporta conocimiento a partir de los significados de los estudiantes en su proceso de práctica pedagógica. De este modo, se busca contribuir a la formación de estudiantes de pedagogía, siendo preciso puntualizar y dejar la reflexión particularmente hacia cinco temas:

En primer lugar, comprender elementos distintivos que develaban los estudiantes al acompañamiento a su praxis pedagógica, ha permitido rescatar de éste, su significativo aporte y sentido a su formación. Se reconoce que la praxis es una experiencia de vida en la que confluyen muchos aspectos, personales y académicos en donde hay urgencias y su reconocimiento y atención resulta vital. De este modo, el cambio de paradigma desde un enfoque de la docencia centrado en la enseñanza a centrarse en el aprendizaje posiciona la actuación del 
docente tutor con capacidad para crear e invitar a oportunidades reales de aprendizaje, generando dispositivos para facilitar que los estudiantes apliquen sus saberes a partir del análisis crítico de la experiencia y así encuentren el sentido de su aprender y de su enseñar. De esta manera, el acompañamiento, el cual centrado en el sujeto estudiante supone un rol más activo a la tutoría, mayor compromiso y responsabilidad.

Segundo, para hacer un correlato entre el proceso teórico y práctico, un elemento crucial en la acción pedagógica es la reflexión, destacándose que las estrategias desplegadas por el modelo de acompañamiento para promover los procesos reflexivos por parte de los estudiantes requieren de actuaciones desafiantes para quienes participan de la praxis pedagógica. Así mismo un enfoque colaborativo hace que la propuesta sea dinámica. La relación pedagógica docentes y estudiantes pretende una praxis atenta al contexto y frente a los cambios, para abrir oportunidades de aprendizaje y potenciar así, la experiencia educativa.

Un tercer tema nos refiere al saber docente, el cual pretende revestirse de un carácter emancipatorio, en este sentido, desde un ser y hacer en diálogo que va adquiriendo un conocimiento a partir de aprendizaje participativo y crítico. Hemos develado, que el saber docente no es sólo tener el conocimiento de la experiencia y ponerlo en práctica sino, por, sobre todo, darle sentido; de esta forma actuar de manera situada reconociéndose y reconociendo complejidades de cada contexto. Esto requiere procesos reflexivos y de investigación, un diálogo entre ambos que promueva la búsqueda de múltiples acciones orientadas a aportar en la formación docente desde perspectivas y frentes, que contribuyan al debate y discusión de problemáticas alojadas en diversas situaciones y contextos.

En relación, y como cuarto tema, el ejercicio de generar conocimiento, es decir que el estudiante reflexione en la acción y con otros, haciendo crítico su acontecer, resulta muy significativo. $\mathrm{Su}$ ejercicio reflexivo en la acción es urgente y abre posibilidades de experiencias nuevas. En este sentido para la formación docente se requiere de diversos sujetos en acción, que contextualicen y que siente las bases para un ejercicio docente en promoción del aprendizaje colaborativo, dialogante y desafiante para un sólido saber pedagógico, siendo por tanto necesario promover vínculos en su acción que invite al estudiante a una oportuna y real experiencia de aprendizaje. 
Como quinto tema, el modelo de acompañamiento y su sistematización abre nuevos campos de indagación en la práctica pedagógica. Esta nos devela un significativo aporte de nuevas propuestas de lectura crítica de la realidad y desafío en la formación inicial docente. A su vez, devela tensiones tanto de la forma como se están llevando a cabo los procesos pedagógicos en las instituciones educativas escolares como a nivel académico y, en este sentido, como nuestro quehacer profesional está implicando en ello. A sí también, los nuevos modelos formativos en pedagogía requieren de un diálogo mancomunado entre los diversos participantes con criterios educativos basales compartidos, para promover un escenario de compromisos reales para el aprendizaje. Este trabajo es un desafío porque no todo está dicho, hay temas que surgen y que nos invitan a reflexionar y analizar ampliamente. Este es un primer acercamiento de lo que se está haciendo con el propósito de hacer una mejor pedagogía que nos convoque a ser más críticos de la experiencia para mejorarla en bien de quienes aprenden. De ahí somos parte.

\section{Notas:}

${ }^{1}$ Nos referiremos a práctica pedagógica a lo que la estudiante realiza en el trabajo pedagógico con niños, niñas, y comunidad en general. Se usará como sinónimo en este trabajo el término praxis. Esto se indica para hacer la distinción del nombre de la asignatura de práctica en centros educativos y/o comunitarios.

${ }^{2}$ Se usará el término "los" para referirse a los y las, esta distinción es sólo para efectos de una lectura más fluida para el lector.

${ }^{3}$ Docente guía se referirá al profesional del centro de práctica y que acompaña de manera directa a estudiante.

${ }^{4}$ Más adelante se mencionará sólo como docente.

${ }^{5} \mathrm{El}$ tutor es el docente el universitario que acompaña el proceso de práctica del estudiante.

${ }^{6}$ Las citas que se presentan en este artículo corresponden a relatos de estudiantes de práctica profesional de la carrera de Educación Parvularia y Básica Inicial que concluye su proceso a inicios del año 2015. Estos relatos de estudiantes de práctica profesional se abrevian como (epp). 


\section{REFERENCIAS BIBLIOGRÁFICAS}

De Souza, J., 2000

Dewey, J., 1998

Freire, P., 1971

Freire, P., 1979

García-Huidobro, J.E., 1998

Ghiso, A., 1998

Jara, O., 1994

Litwin, E., 2008

Martinic, S., 1998

Messina, G., 2003
Sistematización: un instrumento pedagógico en los proyectos de desarrollo sustentable. En: Revista Interamericana de educación de adultos 22. (1, 2 y 3).11-48.

Cómo pensamos, España. Paidós.

Educación como práctica de la libertad. Buenos Aires Siglo XXI. Educación Liberadora. Bilbao. Zero Comentario respecto a la sistematización, en profesionales en la acción. Santiago. Chile. Ediciones CIDE.

De la práctica singular al diálogo con lo plural. Aproximaciones a otros tránsitos y sentidos de la sistematización en épocas de globalización. Lapiragua. Revista Latinoamericana de Educación.

Para sistematizar experiencias: una propuesta teórica y práctica. Sn José. C.R. Centro de estudios y publicaciones, Alforja.

El oficio de enseñar. Condiciones y contextos. Bs. Aires. Paidós

El objeto de la sistematización y sus relaciones con la evaluación y la investigación. Ponencia presentada al Seminario latinoamericano: sistematización de prácticas de animación sociocultural y participación ciudadana en América Latina. Medellín, Fundación Universitaria Luis Amigó-CEAAL, p. 12-14.

La sistematización educativa: acerca de sus especificidades. 
Montero, L., 2001

Perrenoud, P., 2007

Solsona, N., 1999

Schon D., 1983

Torres, A., 2003

Valle, J. y Manso, J., 2011

Zabalza, M. A., 1999
Revista Enfoques Educacionales 6. Departamento de Educación. Facultad de Ciencias Sociales.19-28. La Construcción del conocimiento profesional docente. Buenos Aires: Homo Sapiens.

Desarrollar la práctica reflexiva en el oficio de enseñar. Cap. 2: Saber reflexionar sobre la propia práctica $¿$ es este el objetivo fundamental de la formación de los enseñantes? España. Ed. Grao.

El aprendizaje cooperativo: una estrategia para la comunicación. Aula de Innovación Educativa, 80, 65-67.

The Reflective Practioner. How Professionals think inn action. Nueva York, Basic Books INC Publishers.

Experiencia de sistematización con cinco organizaciones populares. Universidad Pedagógica Nacional, Bogotá.

La nueva formación inicial del profesorado de educación secundaria: modelo para la selección de buenos centros de práctica. EN. Revista Educación N| 354. EneroAbril 2001. La formación práctica en estudiantes universitarios. Repensando el practicum. Ministerio de Educación España. Proceso histórico y personal: de profesor de materia a educadortutor. En P. Arnáiz y J. Riart (Eds.), La tutoría: de la reflexión a la práctica (pp. 33- 48). Barcelona: EUB. 
Zeichner, N., 2010

Rethinking the Connections Between Campus Courses and Field Experiences in College and University-Based Teacher Education. Journal of Teacher Education, 61(1-2), 89-99. 